Bull. Austral. Math. Soc.

VoL. 46 (1992) [385-389]

\title{
ON SUFFICIENCY OF THE KUHN-TUCKER CONDITIONS IN NONDIFFERENTIABLE PROGRAMMING
}

\author{
FUAN ZHAO
}

\begin{abstract}
Some generalised invex conditions are given for a nondifferentiable constrained optimisation problem, generalising those of Hanson and Mond for differentiable problems. Some duality results are obtained.
\end{abstract}

\section{INTRODUCTION}

Let $f, g_{1}, g_{2}, \ldots, g_{m}$ be local Lipschitz functions defined on an open subset $C$ of $R^{n}$. Consider the problem

$$
\operatorname{MIN} f(x) \text { subject to } g_{i}(x) \leqslant 0 \quad(i=1,2, \ldots, m), x \in C .
$$

Several necessary conditions have been established for a local optimal solution of $(P)$ to satisfy Kuhn-Tucker conditions, for example $[6,7,2]$. Denote by $f^{0}(x ; d)$ the Clarke generalised directional derivative of $f$ at $x$ in the direction $d$ (see [2]), and by $\partial f(x)$ the Clarke generalised subgradient of $f$ at $x$. Then assuming a constraint qualification, the Kuhn-Tucker necessary conditions for a minimum at $\bar{x}$ are:

$$
\left(\exists \lambda_{i} \geqslant 0, i=1,2, \ldots, m\right) 0 \in \partial f(\bar{x})+\sum_{i=1}^{m} \lambda_{i} \partial g_{i}(\bar{x}),(\forall i) \lambda_{i} g_{i}(\bar{x})=0
$$

If all the functions are continuously differentiable, then $\partial f(\bar{x})=\{\nabla f(\bar{x})\}$ and $\partial g_{i}(\bar{x})=$ $\left\{\nabla g_{i}(\bar{x})\right\}$. These necessary conditions at a feasible point $\bar{x}$ become also sufficient if all the functions are convex, or under weaker conditions given by Hanson [4], or by Hanson and Mond [5].

\section{Received 13 November 1991}

The author would like to thank Dr. B.D. Craven of the University of Melbourne for his invaluable help in the preparation of this paper.

Copyright Clearance Centre, Inc. Serial-fee code: 0004-9729/92 \$A2.00+0.00. 


\section{A SIMPLE PROOF OF HANSON AND MOND'S THEOREM}

Hanson and Mond [5] say that the objective $f$ and the constraint functions $g_{i}(i=1,2, \ldots, m)$ are Type $I$ invex with respect to $\eta$ at $\bar{x}$ if there exists a function $\eta: R^{n} \rightarrow R^{n}$ such that, for all $x$ feasible for $(\mathrm{P})$,

$$
f(x)-f(\bar{x}) \geqslant[\nabla f(\bar{x})]^{T} \eta(x) \text { and }-g_{i}(\bar{x}) \geqslant\left[\nabla g_{i}(\bar{x})\right]^{T} \eta(x) .
$$

Ben-Israel and Mond [1] have characterised such functions $\eta$.

LEMMA 1. If $v_{0}, v_{1}, v_{2}, \ldots, v_{k}$ are vectors in $R^{n}$, with $k<n$, and $t_{i}(i=0,1, \ldots, k)$ are nonnegative real numbers, then the linear inequality system $v_{i}^{T} x \leqslant t_{i}(i=0,1, \ldots, k)$ has a nonzero solution $x$.

Proof: It is sufficient to prove the case when all $t_{i}=0$. If $k=n-1$ and the $v_{i}$ are linearly independent, then the matrix $M$ whose columns are the $v_{i}$ is invertible. Let $e=(1,1, \ldots, 1)$; then $x=-M^{-1} e \neq 0$ satisfies $(\forall i) v_{i}^{T} x=-1<0$. Suppose that the $v_{i}$ are linearly dependent, say $\bar{v}_{,}=\sum_{j \neq l} \lambda_{j} v_{j}$ for some numbers $\lambda_{j}$. There is a nonzero $x$ satisfying $v_{i}^{T} x=0(i=0,1,2, \ldots, k, i \neq s)$ since $k<n$; then $v_{\lrcorner}^{T} x=0$ also. A nonzero $x$ is found in either case.

THEOREM 2. [5, Theorem 2.2]. If $(P)$ has $k<n$ active constraints at an optimal point $\bar{x}$ for $(P)$, then $f$ and $g_{i}(i=1,2, \ldots, m)$ are Type $I$ invex with respect to a common vector function $\eta \not \equiv 0$.

Proof: At a feasible point $x, t_{0}=f(x)-f(\bar{x}) \geqslant 0$ and each $t_{i}=-g_{i}(\bar{x}) \geqslant 0$. Let $v_{0}=\nabla f(\bar{x})$ and $v_{i}=\nabla g_{i}(\bar{x})$. By Lemma $1,(1)$ has a nonzero solution $\eta \equiv \eta(x)$.

\section{SUFFICIENCY OF THE KUHN-TUCKER CONDITIONS}

If (KT) holds, then

$$
\left(\exists \zeta_{0} \in \partial f(\bar{x}), \zeta_{i} \in \partial g_{i}(\bar{x})\right) \zeta_{0}+\sum_{i>0} \lambda_{i} \zeta_{i} ;(\forall i>0) \lambda_{i} \geqslant 0, \lambda_{i} g_{i}(\bar{x})=0
$$

thus $\lambda_{i}=0$ for inactive constraints. The function $f$ and $g_{i}$ will now be called Type $I$ invex with respect to a vector function $\eta$ at $\bar{x}$ if, for each feasible $x$,

$$
f(x)-f(\bar{x}) \geqslant \zeta_{0}^{T} \eta(x) \text { and }-g_{i}(\bar{x}) \geqslant \zeta_{i}^{T} \eta(x)(i=1,2, \ldots, m) .
$$

Inactive constraints may be omitted from (2).

Theorem 3. Let (KT2) hold at a feasible point $\bar{x}$ of $(P)$, where the number of active constraints is $k<n$. Then $\bar{x}$ is optimal if and only if $f$ and $g_{i}$ are Type $I$ invex with respect to a common vector function $\eta$. 
Proof: If (2) holds, and $x$ is feasible, then

$$
f(x)-f(\bar{x})=f(x)-f(\bar{x})+\sum_{i>0} \lambda_{i}\left(-g_{i}(\bar{x})\right) \geqslant\left(\zeta_{0}+\sum_{i>0} \zeta_{i}\right)^{T} \eta(x)=0
$$

using (KT2) and (3). Conversely, if $\bar{x}$ is a minimum, then (2) is proved in the same manner as Theorem 2.

REMarKs. The first part of this proof does not use the hypothesis $k<n$.

The vectors $\zeta_{0}$ and $\zeta_{i}$ cannot be replaced here by arbitrary elements of $\partial f(\bar{x})$ and $\partial g_{i}(\bar{x})$ respectively, because then $\zeta_{0}+\sum_{i>0} \zeta_{i}$ is no longer zero.

In [3], a generalised invex property was defined in terms of Clarke generalised directional derivatives, namely

$$
f(x)-f(\bar{x}) \geqslant f^{0}(\bar{x} ; \eta(x)), g_{i}(x)-g_{i}(\bar{x}) \geqslant g_{i}^{0}(\bar{x} ; \eta(x))(i=1,2, \ldots, m) .
$$

It was shown in [3] that (KT2) at a feasible point $\bar{x}$, together with (3), imply a minimum at $\bar{x}$. Consider now a weakened version of (3), that for all feasible $x$,

$$
f(x)-f(\bar{x}) \geqslant f^{0}(\bar{x} ; \eta(x)), g_{i}(\bar{x}) \geqslant \quad g_{i}^{0}(\bar{x} ; \eta(x)) \quad(i=1,2, \ldots, m) .
$$

However, (4) is not a consequence of (2), whether or not $k<n$.

THEOREM 4. If (KT2) and (4) hold at a feasible point $\bar{x}$, then $\bar{x}$ is a minimum of $(P)$.

Proof: If $x$ is feasible for (P), then

$$
\begin{aligned}
f(x)-f(\bar{x}) & =f(x)-f(\bar{x})+\sum_{i>0} \lambda_{i}\left(-g_{i}(\bar{x})\right) \\
& \geqslant f^{0}(\bar{x} ; \eta(x))+\sum_{i>0} \lambda_{i} g_{i}^{\circ}(\bar{x} ; \eta(x)) \\
\geqslant & \theta_{0}^{T} \eta(x)+\sum_{i>0} \lambda_{i} \theta_{i}^{T} \eta(x) \\
& \quad \text { for all } \theta_{0} \in \partial f(\bar{x}) \text { and all } \theta_{i} \in \partial g_{i}(\bar{x}) \\
\geqslant & 0 \quad \text { by (KT2), substituting } \theta_{i}=\zeta_{i}(i \geqslant 0) .
\end{aligned}
$$




\section{Subgradient duality}

Schechter $[8,9]$ proposed a dual problem for convex nondifferentiable problems, and proved a subgradient duality result. This dual to $(\mathrm{P})$ has the form

$$
\operatorname{MAX} f(z)+\sum_{i} \theta_{i} g_{i}(z) \text { subject to }(\forall i) \quad \theta_{i} \geqslant 0,0 \in \partial f(z)+\sum_{i} \theta_{i} \partial g_{i}(z) .
$$

If $(z, \theta)$ is feasible for (D), then there exist $\omega_{0} \in \partial f(z)$ and $\omega_{i} \in \partial g_{i}(z)(i>0)$ such that $0=\omega_{0}+\sum_{i>0} \theta_{i} \omega_{i}$. Consider the modified invex property, generalising the Type II invex of Hanson and Mond [5], that for some function $\eta(.,$.$) ,$

$$
f(y)-f(z) \geqslant \omega_{0}^{T} \eta(y, z), \quad(\forall i>0)-g_{i}(z) \geqslant \omega_{i}^{T} \eta(y, z) .
$$

REMARK. The definition in [5] assumes the more restrictive $\eta(z)$.

Theorem 5. Assume that (2) holds whenever $x$ is feasible for $(P)$, and (5) holds whenever is feasible for $(P)$ and $(z, \theta)$ is feasible for $(D)$. Then weak duality holds:

$$
f(x) \geqslant f(z)+\sum \theta_{i} g_{i}(z) \text {. }
$$

If also (KT2) holds for $(P)$ at $\bar{x}$, with multipliers $\lambda_{i}$, then zero duality gap holds: $(\bar{x}, \lambda)$ is feasible for $(D)$, and $f(\bar{x})=f(\bar{x})+\sum \lambda_{i} g_{i}(\bar{x})$.

Proof: Weak duality

$$
\begin{array}{rlrl}
f(x)-\left[f(z)+\sum \theta_{i} g_{i}(z)\right] & \geqslant \omega_{0}^{T} \eta(y, z)-\sum \theta_{i} g_{i}(z) & & \text { by (5) } \\
& =-\sum \theta_{i} \omega_{i}-\sum \theta_{i} g_{i}(z) & & \text { by a constraint of (D) } \\
& \geqslant+\sum \theta_{i} g_{i}(z)-\sum \theta_{i} g_{i}(z) & & \text { by (5) } \\
& =0 . &
\end{array}
$$

Zero duality gap The statements follow from (KT2).

Theorem 6. Let $(\bar{x}, \lambda)$ be feasible for $(D)$, let (5) hold at $y=\bar{x}$, and let $\lambda^{T} g(\bar{x})=0$. Then $(\bar{x}, \lambda)$ is optimal for $(D)$.

PROOF: There are $\omega_{0} \in \partial f(z)$ and $\omega_{i} \in \partial g_{i}(z)$ such that $0=\omega_{0}+\sum_{i>0} \lambda_{i} \omega_{i}$. A similar calculation to the weak duality proof of Theorem 5 shows that, if $(z, \theta)$ is feasible for (D), then

$$
\left[f(\bar{x})+\lambda^{T} g(\bar{x})\right]-\left[f(z)+\theta^{T} g(z)\right] \geqslant \lambda^{T} g(\bar{x})-0 \text { by hypotheses. }
$$




\section{REFERENCES}

[1] A. Ben-Israel and B. Mond, 'What is invexity?', J. Austral. Math. Soc., series B 28 (1968), 1-9.

[2] F.H. Clarke, Nonsmooth analysis and optimization (Wiley, New York, 1983).

[3] B.D. Craven, 'Nondifferentiable approximation by smooth approximations', Optimization 17 (1986), 3-17.

[4] M.A. Hanson, 'On sufficiency of the Kuhn-Tucker conditions', J. Math. Anal. Appl. 80 (1981), 545-550.

[5] M.A. Hanson and B. Mond, 'Necessary and sufficient conditions in constrained optimization', Math. Programming 37 (1987), 51-58.

[6] J.-P. Hiriart-Urruty, 'On necessary optimality conditions in nondifferentiable programming', Math. Programming 14 (1978), 73-86.

[7] J.-P. Hiriart-Urruty, 'Refinements of necessary optimality conditions in nondifferentiable programming', Appl. Math. Optim. 5 (1979), 63-83.

[8] M. Schechter, 'A subgradient duality theorem', J. Math. Anal. Appl. 61 (1977), 850-855.

[9] M. Schechter, 'More on subgradient duality', J. Math. Anal. Appl. 71 (1979), 252-262.

Institute of Applied Mathematics

Academia Sinica

Beijing 100080

Peoples Republic of China 